\title{
浅析高中生物课堂教学中的有效提问
}

\author{
黄玉琼 \\ 江西省赣州市赣县第三中学 \\ DOI:10.32629/jief.v2i9.1701
}

[摘 要] 在高中生物教学中, 很多学生缺乏兴趣, 导致课堂效率低下, 而提问作为课堂教学中师生互动或生生互动的主要形式, 可以使教师 将关注的焦点从教师转移到学生身上。有效提问意味着教师所提出的问题能够引起学生的回应或回答, 且这种回应或回答让学生更积极地参 与学习过程。笔者结合自身教学实际，从提问的针对性、把握提问的时机以及难度，抓住兴趣点提问几个方面浅谈自己的看法。

[关键词] 有效提问; 高中生物; 课堂教学

中图分类号: G633.91 文献标识码: A

教育心理学告诉我们: 学生的思维过程往往是从问题开始, 古语亦 云: 学起于思, 思源于疑。有经验的老师在教学过程中, 总是精心设计 提问, 意图点燃学生思维的火花, 激发他们的探索欲望, 并有意识地为 他们发现疑难、解决疑难提供桥梁和阶梯, 引导他们不断攀登知识的高 峰。但是, 并不是所有的课堂提问都能达到预期的目标, 有的提问零敲 碎打、毫无联系, 有的提问肤浅平庸、单调陈旧。这些置学生于被动地 位的提问, 抑制了学生的思维活动, 与开发学生智能目标背道而驰。我 们在日常课堂教学活动中应怎样做才能达到提问的有效性? 怎样的有效 提问才能真正引导学生主动参与学习? 下面就根据笔者生物教学来谈谈 几点粗浅的看法。

\section{1 提问应有针对性}

\section{1 针对教材实际提问}

教师教师在充分解读教材, 了解教学的重点、难点的基础上, 紧紧 围绕教材的训练重点, 突破难点, 问到关键之处。例如, “基因突变” 这 一节内容的教学重难点是基因突变的意义, 所以可以设置以下问题: (1) 基因突变能产生新的基因吗? (2)这些新的基因产生的新性状对生物的生 存有什么意义? (3)自然环境会选择哪些个体生存下来? (4)基因突变对生 物的进化有何意义? 这几问环环相扣, 层层相接, 在有限的时间里, 抓 住了重点, 突出要害。

\section{2 针对学生实际提问}

对不同基础的学生应有所区别, 因人而异。对优等生, 可提一些综 合应用、分析理解、总结评价之类的认知性问题, 使其自感不足, 形成 一定压力; 对基础较差的学生, 则可提一些模式识制、知识回忆、形成 联系之类的记忆性问题, 并且适当时给以引导和补充, 使其增加信心。 例如, 在 “杂交育种原理及应用” 这一内容的教学中笔者设置了以下问 题: (1)杂交育种的原理是什么? (2)杂交育种的优点和不足有哪些? (3)你 能阐述杂交育种的细胞学理论基础吗? (4)杂交育种技术为什么耗时相对 较长? 杂交育种能产生一些优良的作物新品种, 那么它能产生一些新性 状吗? 第一、二个问题主要考查学生记忆能力, 提问水平低, 可让基础 较差的学生回答; 而第三、四两题考查学生对知识原理理解的深度以及 综合分析能力, 如第三个问题学生要理解减数分裂中非同源染色体的自 由组合导致基因重组这一细胞学基础才能正确回答, 对学生的水平要求 及思维层次都比较高, 可让认知水平较高的学生回答。

\section{2 控制课堂提问的时机}

2.1 了解学生疑难之处所在而提出问题

如学生没有疑问则设置疑点, “制造”矛盾, 以打开学生心灵的门扉, 激发他们去思考, 逐步引入。在 “细胞呼吸” 这一内容的教学中, 提出 问题: 同学是否知道呼吸和细胞呼吸的区别, 是否能将呼吸和细胞呼吸 等同。通过回忆比较, 学生得出其实呼吸是人和动物体与外界进行的气 体交换过程。而细胞呼吸是指有机物在细胞内经过一系列的氧化分解, 生成二氧化碳或其他产物, 释放出能量并生成 ATP 的过程。呼吸和细胞 呼吸的现象和本质是有所区别的。通过制造了呼吸和细胞呼吸这对矛盾
提问, 学生更深刻地理解了细胞呼吸的含义。笔者在平时经常积累学生 易错的问题, 具有探究因素的问题, 把它作为打开大门登堂入室的钥匙, 激起学生思维的波涛, 引导学生进入探究。讲课过程中插入这类问题, 加强了学生脑力活动的强度, 也使上课波澜起伏, 增强效果。

2.2 在新旧知识的结合处提问

例如在光合作用学习时, 教师可以设计这样一个问题: 假如你是从 事农业种植工作的, 请结合光合作用的原理和生产实际来阐述在种植过 程中应采取的一些措施, 并说明理由。这个问题一提出, 同学各抒已见, 有的同学结合光合作用的化学方程式考虑光合作用的原料问题; 有的结 合植物矿质元素来考虑施肥问题; 有的结合对光能利用特点考虑光的适 当补充问题; 更有同学结合学习过的酶的知识, 联系温度对酶的影响来 考虑环境温度的控制, 结合自然环境下温度控制的难度联系到大棚种植 等一系列问题。通过提问使学生了解知识是如何深化发展的, 沟通新旧 知识的联系, 并学会了分析、比较等思维方法。

2.3 在学生的思维受阻处提问

学生在学习由不知到知, 从不懂到会, 出现思维疑难或思维受阻是 经常发生的。因此, 这就需要教师根据学生的实际情况, 灵活处置, 随 时调整或改变原来准备的问题, 把问题提在 “火候” 上。例如: 在 “细 胞分化” 的教学中, 学生对 “全能性” 的学习出现 “卡壳”, 所以笔者将 原来的自己讲解改为以下几个问题的设置: (1)分化了的细胞具有和受精 卵相同的遗传物质, 是否能像受精卵一样能发育成完整个体? (2)理论上 动物细胞与植物细胞全能相似吗? 为什么? (3)实际上动物体细胞能否表 现出全能性? 如果不用提问的方式, 而是由教师平铺直叙讲解, 效果显 然就差多了。

\section{3 抓住兴趣点提问}

所谓兴趣点, 就是能够激发学生学习兴趣, 促进学生思考理解的知 识点。抓住知识点提问, 可以激起学生的求知欲望, 使学生的情绪处于 积极六奋状态, 激发学生寻找正确答案的积极性。尤其在学习新知识之 前, 教师有意识地提出问题, 激发学生学习兴趣, 以营造生动愉快的教 学情境, 引导学生带着浓厚的学习兴趣去积极思维, 寻求新的知识。如 在教学 “伴性遗传” 时, 笔者设计了以下问题 (1)动物为什么有雌雄, 人 类为什么有男女? (2)为什么人类的秃顶性状多在男性表现? (3)为什么禁 止近亲结婚? 几个问题一提出, 课堂气氛顿时活跃起来, 这样就能使学 生在轻松愉快的气氛中进入探求新知的阶段。

\section{[参考文献]}

[1]李万清. 高中生物教学中科学探究能力的培养策略[J]. 课程教育 研究,2019(19):169-170.

[2]言冠颖. 高中生物学教学培养学生科学探究能力的策略调查与设 计[D].2019.

[3]张云. 高中生物教学中发展学生核心素养的实践与思考[J].试题 与研究,2020(24):195. 